\title{
BMJ Open Automated oxygen administration versus conventional oxygen therapy after major abdominal or thoracic surgery: study protocol for an international multicentre randomised controlled study
}

Erwan L'her, ${ }^{1,2}$ Samir Jaber, ${ }^{3}$ Daniel Verzilli, ${ }^{3}$ Christophe Jacob, ${ }^{4}$ Brigitte Huiban, ${ }^{4}$ Emmanuel Futier, ${ }^{5}$ Thomas Kerforne, ${ }^{6}$ Victoire Pateau, ${ }^{2,7}$ Pierre-Alexandre Bouchard, ${ }^{8}$ Maellen Gouillou, ${ }^{9}$ Emmanuel Nowak, ${ }^{9}$ François Lellouche ${ }^{8}$

To cite: L'her E, Jaber S, Verzilli D, et al. Automated oxygen administration versus conventional oxygen therapy after major abdominal or thoracic surgery: study protocol for an international multicentre randomised controlled study. BMJ Open 2019;9:e023833. doi:10.1136/ bmjopen-2018-023833

Received 25 April 2018 Revised 8 August 2018 Accepted 20 October 2018
Check for updates

(C) Author(s) (or their employer(s)) 2019. Re-use permitted under CC BY-NC. No commercial re-use. See rights and permissions. Published by BMJ.

For numbered affiliations see end of article.

Correspondence to Professor Erwan L'her; erwan.Iher@chu-brest.fr

\section{ABSTRACT}

Introduction Hypoxemia and hyperoxia may occur after surgery with potential related complications. The Free $\mathrm{O}_{2}$ Post0p trial is a prospective, multicentre, randomised controlled trial that evaluates the clinical impact of automated $\mathrm{O}_{2}$ administration versus conventional $\mathrm{O}_{2}$ therapy after major abdominal or thoracic surgeries. The study is powered to demonstrate benefits of automated oxygen titration and weaning in term of oxygenation, which is an important surrogate for complications after such interventions.

Methods and analysis After extubation, patients are randomly assigned to the Standard (manual $\mathrm{O}_{2}$ administration) or $\mathrm{FreeO}_{2}$ group (automated closed-loop $\mathrm{O}_{2}$ administration). Stratification is performed for the study centre and a medical history of chronic obstructive pulmonary disease (COPD). Primary outcome is the percentage of time spent in the target zone of oxygen saturation, during a 3-day time frame. In both groups, patients will benefit from continuous oximetry recordings. The target zone of oxygen saturation is $\mathrm{SpO}_{2}=88 \%-92 \%$ for patients with COPD and $92 \%-96 \%$ for patients without COPD. Secondary outcomes are the nursing workload assessed by the number of manual $\mathrm{O}_{2}$ flow adjustments, the time spent with severe desaturation $\left(\mathrm{SpO}_{2}<85 \%\right)$ and hyperoxia area $\left(\mathrm{SpO}_{2}>98 \%\right)$, the time spent in a hyperoxia area $\left(\mathrm{SpO}_{2}>98 \%\right)$, the $\mathrm{VO}_{2}$, the duration of oxygen administration during hospitalisation, the frequency of use of mechanical ventilation (invasive or non-invasive), the duration of the postrecovery room stay, the hospitalisation length of stay and the survival rate.

Ethics and dissemination The Free $\mathrm{O}_{2}$ PostOp study is conducted in accordance with the declaration of Helsinki and was registered on 11 September 2015 (http://www. clinicaltrials.gov). First patient inclusion was performed on 14 January 2016. The results of the study will be presented at academic conferences and submitted to peer-reviewed journals.

Trial registration number NCT02546830.
Strengths and limitations

The Free $0_{2}$ Post0p trial is the first and largest clinical evaluation of automated oxygen titration over standard of care in patients undergoing major surgeries.

- As automated oxygen titration is not the standard of care for postoperative patients, the study was designed as a superiority study.

- The use of continuous and non-averaged $\mathrm{SpO}_{2}$ values monitoring in all groups of patients enables precise oxygenation evaluation during the entire study period.

- One limitation of the study is that investigators are aware of the inclusion group, while blinding is difficult in studies with respiratory support.

- Second limitation could be that the oxygenation status assessment would have been more precise using arterial blood gases.

\section{INTRODUCTION}

Background and rationale

Current standards for prescribing oxygen recommend providing adequate flows to correct hypoxemia and avoid hyperoxia. ${ }^{12}$ While deleterious effects of hypoxemia are well known, the potential harmful effects of hyperoxia are underappreciated. Yet, hyperoxia may increase mortality in patients with severe chronic obstructive pulmonary disease $(\mathrm{COPD})^{3-5}$ and may cause cardiac and neurological adverse toxicities in certain situations. ${ }^{6-8}$ Precise control of $\mathrm{O}_{2}$ flows is difficult to achieve in clinical practice and is time-consuming. ${ }^{9}$

The FreeO ${ }_{2}$ system (Oxynov, Quebec, Canada) is an innovative device, developed in 
collaboration between our researchers from Brest-France, Quebec-Canada University Hospitals and Oxynov Inc., an R\&D spin-off from Laval University-Quebec. FreeO ${ }_{2}$ is a closed-loop device that automates oxygen administration to spontaneously breathing patients in response to pulse oximetry $\left(\mathrm{SpO}_{2}\right)$ continuous measurements. ${ }^{10}$ Automated $\mathrm{O}_{2}$ administration allows to maintain constant $\mathrm{SpO}_{2}$ within a predetermined range using variable $\mathrm{O}_{2}$ flows, as opposed to manual $\mathrm{O}_{2}$ administration where the flow is kept constant, with variable $\mathrm{SpO}_{2}$ values. In preterm infants receiving mechanical ventilation, automated $\mathrm{O}_{2}$ control results in more time spent within the intended $\mathrm{SpO}_{2}$ target. ${ }^{11-13}$ In a healthy adult model with induced hypoxemia, such a system was more efficient to maintain $\mathrm{SpO}_{2}$ within the oxygenation target, while ensuring a significant reduction of hypoxemia and hyperoxia periods, as compared with constant $\mathrm{O}_{2}$ flows. ${ }^{14}$ Its efficacy has also been validated in hospitalised patients with $\mathrm{COPD}^{15}$ or during the early emergency care of patients with acute respiratory distress. ${ }^{10}$

Following major abdominal or thoracic surgery, the risk of hypoxemia may be high while considering patients' clinical status (obstructive sleep apnoea (OSA), restrictive pathologies related to obesity, frequent comorbidities), the type of surgery and anaesthesia. ${ }^{16-21}$ Hypoxemia may occur either during the immediate postoperative period (they are mainly related to surgery or anaesthesia) or may be delayed up to 3 days without clear trigger or underlying pathologies. The potential interest of the FreeO $_{2}$ system, using artificial intelligence closed-loop adjustments and predictive analytics, will thus be (1) to perform frequent and rapid $\mathrm{O}_{2}$ adjustments in response to oxygenation condition variations (up to each second), or to any physiological condition changes (movement, speech, eating, toilet); (2) to enable remote monitoring and data recording in isolated clinical settings (ie, non-ICU surgical ward), in order to detect clinical deterioration at a very early stage through integration and fusion of informations; (3) to avoid maintenance of unnecessary high $\mathrm{O}_{2}$ flow that may be potentially deleterious (hypercapnia worsening, coronary and/or cerebral artery vasoconstriction, etc).

\section{Objectives}

The aim of the study is to assess the use feasibility of the $\mathrm{FreeO}_{2}$ system so as to deliver oxygen automatically in the postanaesthesia care unit in a patient population admitted for major abdominal and thoracic surgery. Our hypothesis is that the $\mathrm{FreeO}_{2}$ system will provide a better control of the oxygen saturation and reduce postoperative hypoxemia.

\section{Trial design}

The $\mathrm{FreeO}_{2}$ PostOp study is an investigator-initiated, prospective, multicentre, randomised, controlled open trial on medical devices comparing two strategies of oxygen therapy following major surgeries with either standard treatment or automated closed-loop oxygenation.
Patients are randomly assigned to the Standard (manual $\mathrm{O}_{2}$ administration) or $\mathrm{Free} \mathrm{O}_{2}$ group (automated closedloop $\mathrm{O}_{2}$ administration).

\section{METHODS: PATIENTS, INTERVENTIONS AND OUTCOMES Study setting}

The $\mathrm{FreeO}_{2}$ PostOp study is taking place in five different university hospitals in France and Canada (Brest, Clermont-Ferrand, Montpellier, Poitiers, Quebec). The entire study will be performed in accordance with the International Conference on Harmonisation and Good Clinical Practice guidelines. $^{22}$

\section{Eligibility criteria}

Inclusion criteria

To be included, adult patients ( $\geq 18$ years) may be screened for scheduled abdominal or thoracic surgery during the anaesthesia consultation and be considered as requiring general anaesthesia with an expected duration of 2 hours or more in the participating centres.

Patients with intermediate to high risk for postoperative pulmonary complications following abdominal surgery with general anaesthesia are eligible for participation. To identify such patients, the ARISCAT risk score will be used. ${ }^{23}$ An ARISCAT risk score $\geq 26$ is associated with an intermediate to high risk for postoperative pulmonary complications.

Informed consent is to be signed before the surgery (figure 1).

\section{Exclusion criteria}

Patients fulfilling one or more of the following criteria will not be included: life-threatening condition requiring an unplanned emergent surgery, lack of informed consent prior to randomisation, non-adult patients (age $<18$ years), patients with a Body Mass Index $\geq 35 \mathrm{~kg}$ / $\mathrm{m}^{2}$, patients with OSA, pregnant or lactating women, perturbed or non-cooperative patients, to provide a relatively homogeneous study population and avoid potential confounding factors in the interpretation.

\section{Randomisation criteria}

Patients will be randomised if they fulfil all the following criteria: availability of the $\mathrm{FreeO}_{2}$ prototype; absence of criteria of severity justifying the immediate use of ventilatory support (loss of consciousness with a Glasgow Coma Score $\leq 12$ ), serious ventricular rhythm disorders, haemodynamic instability (systolic blood pressure $<80 \mathrm{~mm}$ $\mathrm{Hg}$ or recourse to vasopressors), cardiac or respiratory arrest, $\mathrm{pH}<7.35$ and $\mathrm{PaCO}_{2}>55 \mathrm{~mm} \mathrm{Hg}$ (if measured), and necessity of an $\mathrm{O}_{2}$ flow less than $15 \mathrm{~L} / \mathrm{min}$ to maintain a $\mathrm{SpO}_{2}$ higher than $92 \%$; no emergent surgery required for an adverse event; pulse oximetry signal is available.

\section{Study intervention}

Patients eligible for randomisation will be randomly assigned to the Standard or $\mathrm{Free}_{2}$ group (figure 2). The maximal study duration is 3 days. The study was stopped 


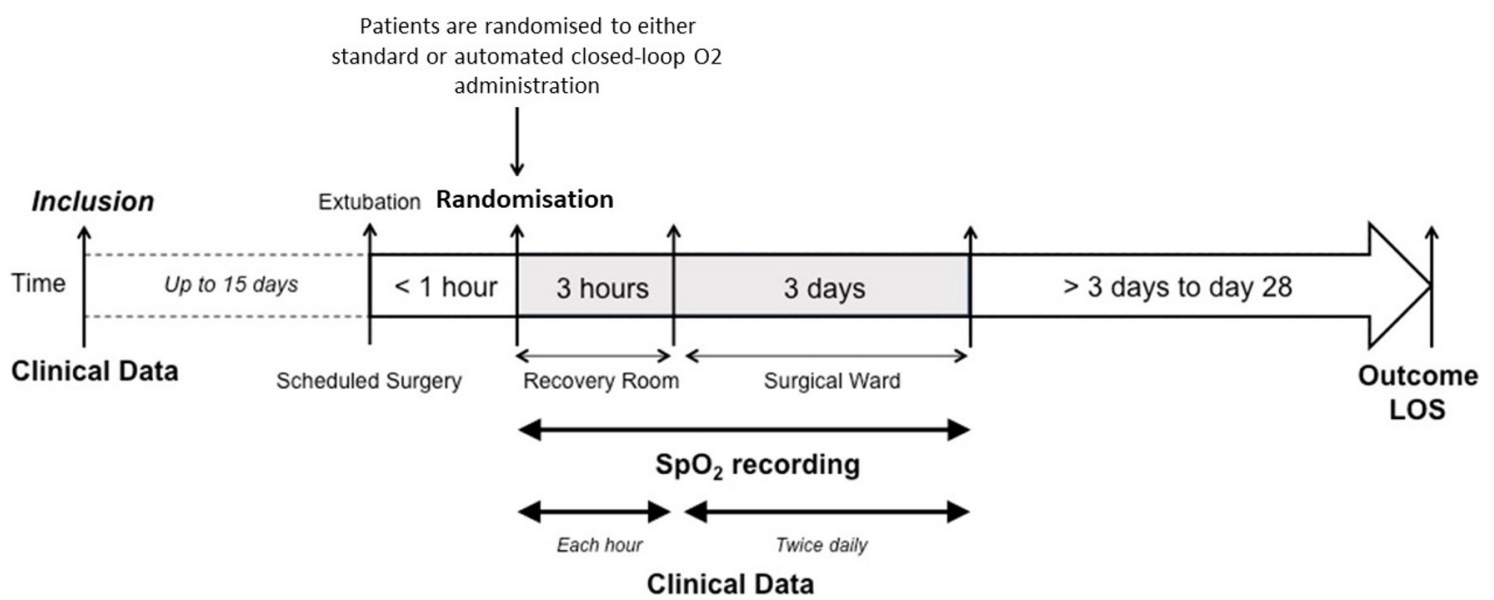

Figure 1 Participant timeline of the $\mathrm{FreeO}_{2}$ PostOp study. Inclusion is performed during the anaesthesia consultation, prior to a scheduled major surgery ( $\geq 2$ hours' duration; ARISCAT score $\geq 26$ ). Eligibility criteria are verified and the patient needs to sign the informed consent. Randomisation is performed no later than 1 hour following extubation in the recovery room. Clinical data will be recorded each hour during the first 3 hours of care and twice daily for up to 3 days. Continuous $\mathrm{SpO}_{2}$ recording will be performed in each randomisation groups for up to 3 days. Outcome parameters will be assessed at day 28 . LOS, length of stay (days); $\mathrm{SpO}_{2}$, pulse oximetry (\%).

before 3 days if patient was discharged earlier from hospital or in case of discomfort while keeping nasal prongs and sensors.

Automated $\mathrm{O}_{2}$ administration is performed using the FreeO $_{2}$ system (Oxynov) that is set to maintain $\mathrm{SpO}_{2}$ between $92 \%$ and $96 \%$ for patients without COPD or between $88 \%$ and $92 \%$ for patients with COPD. FreeO ${ }_{2}$ is equipped with a $\mathrm{SpO}_{2}$ monitor and an electronically controlled valve that automatically adjusts $\mathrm{O}_{2}$ flows from 0 to $20 \mathrm{~L} / \mathrm{min}$ on a per-second basis, with a $0.1 \mathrm{~L} /$ min precision, according to a closed-loop algorithm in order to reach the predetermined $\mathrm{SpO}_{2}$ target. ${ }^{14}$ Conventional $\mathrm{O}_{2}$ is administered using manual flowmeters, according to standard procedures. All participating units were encouraged to use the same standardised $\mathrm{SpO}_{2}$ target as in the automated $\mathrm{O}_{2}$ administration group, as recommended in international guidelines. ${ }^{12}$ In both arms, oxygen can be administered either using nasal prongs for low flow $\left(\mathrm{O}_{2}<6 \mathrm{~L} / \mathrm{min}\right)$ or standard face mask in all cases $\left(\mathrm{O}_{2}=0-20 \mathrm{~L} / \mathrm{min}\right)$.

Continuous oximetry recordings are performed in each group during the 3 days of the study, using the FreeO monitoring system connected to Nonin 6000 CA flexible adult single-use digital sensors. Position of the sensors is to be controlled at least every 12 hours. In the FreeO ${ }_{2}$ group, the $\mathrm{FreeO}_{2}$ will be used both for oxygen administration and for recording. In the Standard group, the FreeO $_{2}$ will be used in the recording only and the oxygen administration will be set manually.

\section{Standard procedures}

Considering the variety of pathological cases for patients attending the recovery room, medical treatment, including the other respiratory support, is determined by the attending physicians based on clinical needs assessment. All other aspects of patient care after inclusion in the study, including fluid administration, prophylactic antibiotics and postoperative pain management, are made at clinicians' discretion based on the expertise of the staff at each centre and routine clinical practice.

\section{Primary outcome measure}

The primary outcome measure will be the percentage of time spent in the target zone of oxygen saturation, during a 3-day time frame. In both groups (Standard and Free $\mathrm{O}_{2}$ ), patients will be connected to the $\mathrm{FreeO}_{2}$ system to enable continuous oximetry recordings of one $\mathrm{SpO}_{2}$ value per second, either in the recording mode for patients assigned to the Standard group or in the automated closed-loop mode for patients assigned to the $\mathrm{Free}_{2}$ group.

The target zone of oxygen saturation is $\mathrm{SpO}_{2}=88 \%-$ 92\% for patients with COPD and 92\%-96\% for patients without COPD.

\section{Secondary outcome measures}

Several secondary outcome measures will be evaluated during a 3-day time frame: nursing workload assessed by the number of manual $\mathrm{O}_{2}$ flow adjustments and airway management procedures (twice daily assessment); time spent with severe desaturation $\left(\mathrm{SpO}_{2}<85 \%\right)$; time spent with hyperoxia $\left(\mathrm{SpO}_{2}>98 \%\right) ; \mathrm{VO}_{2}$ measured at the end of administration. All data related to oxygenation will be recorded in both groups using the $\mathrm{FreeO}_{2}$ device, enabling us to qualify desaturation using the Oxygen Desaturation Index at different levels (ODI2\% and ODI $4 \%$ ).

Other outcome measures will be assessed during a maximal 28-day time frame: duration of oxygen administration during hospitalisation, number of complications related to the administration of oxygen; frequency of use of ventilation (invasive or non-invasive); duration of hospitalisation; survival rate. 


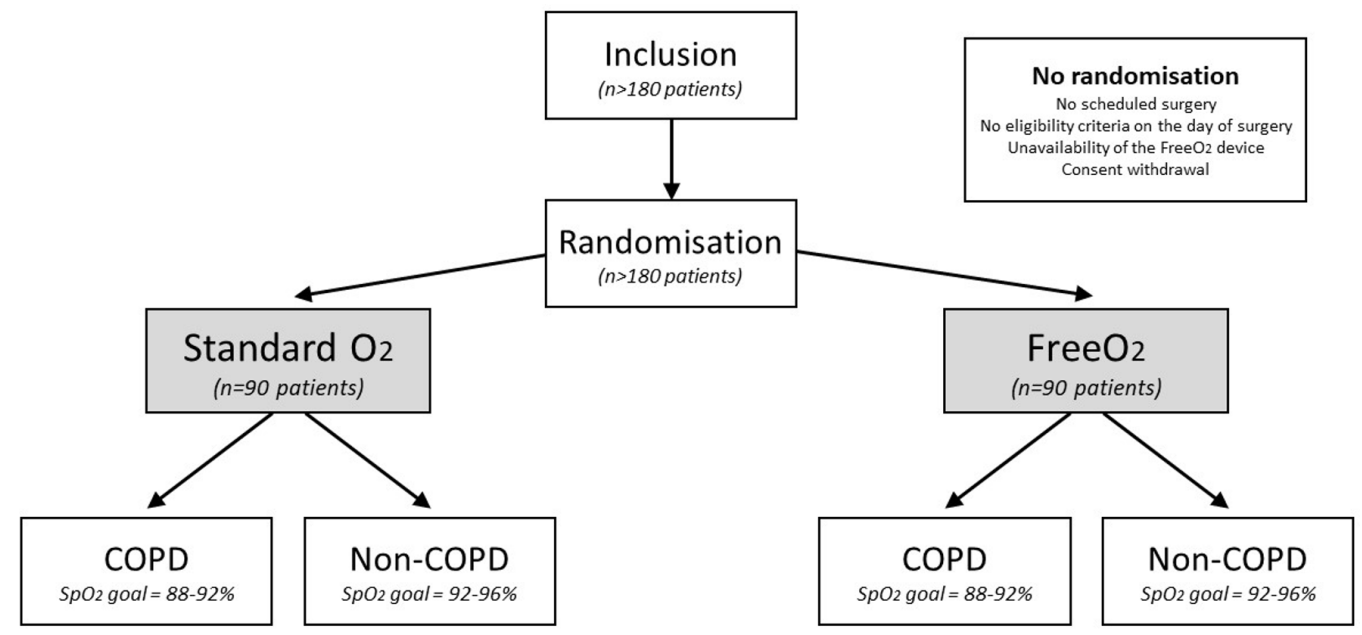

Analysis

$\mathrm{SpO}_{2}$ recordings at $\mathrm{H} 3$ and day 3

Clinical recordings at $\mathrm{H} 3$, day 3 and day 28

Outcome parameters at day 28

Figure 2 Consort diagram of the $\mathrm{FreeO}_{2}$ PostOp study. Randomisation will be performed after verification of the eligibility criteria and it will be stratified according to the presence of a medical history of chronic obstructive pulmonary disease (COPD). For patients without COPD, the target will be a $\mathrm{SpO}_{2}$ range $=92 \%-96 \%$; for patients with COPD, the target will be a $\mathrm{SpO}_{2}$ range $=88 \%-92 \%$. Patients will be assigned either to standard continuous $\mathrm{O}_{2}$ administration (Standard: manual adjustment) or automated closed-loop $\mathrm{O}_{2}$ administration (FreeO $\mathrm{O}_{2}$ : automated adjustment, up to each second); in both groups, continuous $\mathrm{SpO}_{2}$ recordings will be performed during up to 3 days according to the $\mathrm{FreeO}_{2}$ system. $\mathrm{SpO}_{2}$, pulse oximetry (\%).

\section{Participant timeline}

Figure 1 shows the participant timeline of the $\mathrm{FreeO}_{2}$ PostOp study.

\section{Sample size}

Based on previous studies and data from the literature, ${ }^{1020}$ we estimated an $85 \%$ time within oxygenation range with the automated closed-loop oxygen administration system $\left(\right.$ Free $\mathrm{O}_{2}$ ) during the 3 days following surgery and a SD equal or more than $30 \%$. A total number of 180 patients will be needed to demonstrate a $15 \%$ decrease in absolute difference between the Standard and $\mathrm{FreO}_{2}$ groups (90 patients in each group). The $\alpha$ risk is $5 \%$ and the $\beta$ risk is $10 \%$ with a bilateral formulation. The expected duration of patient enrolment will be 3 years.

\section{METHODS: ASSIGNMENT OF INTERVENTION Allocation and sequence generation}

A computer-generated randomisation is performed in the postrecovery room within a 1-hour delay after endotracheal extubation. It is performed using random blocks in a 1:1 ratio, with the use of a centralised web-based management system (Clinfile). Stratification is performed either according to the study centre and a medical history of COPD. After randomisation, treatment is to be initiated within less than 1 hour.

\section{Blinding}

Although the individual study assignments of the patients will not be masked, the coordinating centre and all the investigators will remain unaware of the study group outcomes until the data will be locked.

\section{METHODS: DATA COLLECTION, MANAGEMENT AND ANALYSIS Data collection and management}

At the time of enrolment, physiological characteristics, coexisting medical conditions, surgery and anaesthetics characteristics, and oxygen flow rates are recorded. In both groups, pulse oximetry, respiratory and heart rate are continuously monitored using a dedicated software enabling data extraction from the $\mathrm{FreeO}_{2}$ device. Each hour during the first 3 hours following randomisation and daily during the following 3 days, all standard clinical parameters are collected. Evolution and clinical outcomes are monitored at day 28.

Data are collected and recorded on an electronic case-report form (e-CRF) by a trained investigator or research assistant at each centre. A blank copy of the e-CRF can be printed from the e-CRF; this enables the investigator or research assistant to fill it out with the data of the included patients, which will be captured. Once data collection has been completed, the investigator or research assistant shall sign and date the copy. This document will constitute an integral part of the patient's medical records; as such, it shall be retained permanently. Data recorded in the e-CRF that originate in source documents must be consistent with each other; if they are not, the differences have got to be justified and documented.

\section{Statistical methods}

All the analyses will be performed by the study statistician.

A predefined statistical analysis plan will be followed. The analysis will be performed on an intention-to-treat basis after validation by a blind review committee of the inclusion and exclusion criteria for each patient. All the 
analyses will be conducted by the biostatistics department of the Centre d'Investigation Clinique from Brest University Hospital (CIC INSERM 1412) using SAS V.9.3 statistical software (SAS Institute, Cary, North Carolina, USA). A two-tailed $p$ value equal or less than 0.05 will be considered as statistically significant.

\section{Descriptive analysis of patient groups at baseline}

The continuous variables will be analysed using standard parameters (median, IQRs and extreme values, or mean and SD), while indicating the number of missing data. The category variables will be presented in the form of absolute frequency and percentage, in each treatment group.

The criteria of subject selection will be verified based on the data recorded in the electronic case reports. Falsely included subjects such as those lost to follow-up will be described. Deviations from the protocol will be described and analysed on a case-by-case basis.

\section{Analysis pertaining to the main criteria of evaluation}

The percentages of time within the considered $\mathrm{SpO}_{2}$ range will be compared between the two groups by means of a variance analysis, according to stratification.

\section{Analysis pertaining to the secondary criteria of evaluation}

Secondary criteria of evaluation will be compared between the two treatment groups by means of Student's t-test (or the Mann-Whitney $U$ test, if necessary) for continuous quantitative variables and by means of the $\chi^{2}$ test (or Fisher's exact test) for qualitative variables.

\section{METHODS: MONITORING \\ Data monitoring}

Before starting patient enrolment, all physicians and other healthcare workers in the ICU attend formal training sessions on the study protocol and data collection.

An investigator at each centre is responsible for daily patient screening, enrolling patients in the study, ensuring adherence to the protocol and completing the e-CRF. Research assistants regularly monitor all the centres on site to check adherence to the protocol and the accuracy of the data recorded.

\section{Harms}

The investigator is allowed to temporarily or permanently discontinue the participation of a patient in the study for any reason that would optimally serve the interests of the subject, particularly in case of serious adverse events suspected to be associated with the type of oxygenation method that is used.

\section{Auditing}

The trial is overseen by a steering committee and an independent safety monitoring board composed of three independent experts (Jean-Pierre Frat, Christophe Guitton, Alain Mercat). All centres are monitored by the promoter to check adherence to the protocol and accuracy of recorded data. An investigator at each centre is responsible for enrolling patients and ensuring adherence to the protocol. Research assistants at each centre are responsible for patients' follow-up and for completing the e-CRF.

\section{ETHICS AND DISSEMINATION}

The French national agency for drug and biomedical devices security (ANSM) approved the use of the FreeO $\mathrm{O}_{2}$ device within this study on 5 August 2014. The main modification of the protocol was made after obtaining CE marking for the device (protocol version no. 3); other amendments were related to investigators' changes and an extension of the study length (final version: no. 5). The study sponsor notified all amendments to investigators.

\section{Consent or assent}

The patient is included after having provided a written informed consent to the investigator according to the decision of the central ethics committee. If the patient is not able to understand the information given, he/she can be included if the same procedure is completed with a next of kin. After the patient's recovery, he/she will be asked if he/she agrees to continue the trial.

\section{Patient and public involvement}

Patients and the public were not involved in the design or conduct of the study. There is no plan to disseminate the results to study participants.

\section{Confidentiality}

Data will be handled according to French law. All original records will be archived at trial sites for 15 years. The clean database file will be anonymised and kept for 15 years.

\section{Dissemination policy}

The protocol is reported according to the SPIRIT guidelines. ${ }^{24}$ Findings will be submitted to peer-reviewed journals and presented at local, national and international meetings and conferences according to CONSORT guidelines ${ }^{25}$ to publicise and explain the research to clinicians, commissioners and service users.

Authorship eligibility guidelines will take into account participation to the protocol design and writing, final analysis of the results, patients' recruitment and final manuscript revision.

\section{DISCUSSION}

In postoperative patients, pulmonary function is markedly altered both by general anaesthesia, mechanical ventilation and surgery. Postoperative respiratory complications following surgery are the second most frequent complications after surgery ${ }^{19}$ and considered as a major cause of morbidity and mortality. ${ }^{172627}$ The FreeO ${ }_{2}$ PostOp trial is the first clinical evaluation of automated oxygen titration in patients undergoing major abdominal or thoracic 
surgeries. The objectives of the study are to compare the oxygenation parameters (time in the oxygenation target, time with hypoxemia and with hyperoxia) with usual oxygen therapy and automated oxygen titration and weaning.

\section{Risk and consequences of hypoxemia}

There is a good level of evidence and good acceptation by the medical community that hypoxemia is harmful, ${ }^{28}$ especially in adult patients with myocardial ischaemia ${ }^{29}$ and neurotrauma. ${ }^{30}$ Data also suggest that even short periods of hypoxemia may promote significant negative haemodynamic effects. ${ }^{31}$ In an animal model, right ventricular dilation was observed with only 2 hours of daily hypoxemia. ${ }^{32}$

During the postoperative period, the occurrence of marked oxygen desaturation related to periodic apnoea and hypoventilation for several hours has been recognised for a long time ${ }^{33} 34$ with potentially severe consequences such as myocardial ischaemia. ${ }^{35}$ The incidence of hypoxemia is high in the recovery room $(10 \%-50 \%),{ }^{20}$ and up to $50 \%$ of postoperative patients will demonstrate episodic hypoxemia in the absence of $\mathrm{O}_{2}$ therapy. ${ }^{36}{ }^{37}$ It has also been shown that desaturation episodes are more frequent on the first night after surgery, but may also worsen 3-5 days postoperatively, especially in patients with OSA. ${ }^{38-40}$

Pathophysiology of these $\mathrm{O}_{2}$ desaturations is complex but may be due either to the patient's condition itself (advanced age, COPD, diabetes, obesity, etc), respiratory mechanics modifications (reduction of functional residual capacity, atelectasis, thoracoabdominal compliance decrease), but also to the use of pharmaceutical agents that are given during surgery (anaesthetics and neuromuscular blocking drugs) or those that are given to relief postoperative pain (opioids and sedatives). ${ }^{34}$

Our hypothesis is that desaturations will be reduced with the automated oxygen titration (FreeO ${ }_{2}$ group), as the oxygen flow is titrated every second and that desaturations will immediately lead to increasing of oxygen flowrate. In previous studies comparing manual and automated oxygen administration, it was demonstrated that time with hypoxemia was reduced with FreeO ${ }_{2}{ }^{10} 15$ However, no data are available during the postoperative care.

\section{Automated weaning of oxygen}

Oxygen supplementation has initially been promoted to decrease postoperative hypoxemia, even if it is also known that it will not have any effect on the overall number of central or obstructive apnoea, neither atelectasis. ${ }^{33}$ Not all patients will benefit from systematic $\mathrm{O}_{2}$ administration, ${ }^{41}$ but probably only specific patients with a highrisk profile, especially those following long duration and major thoracic or abdominal surgery. ${ }^{42-44}$ Moreover, standard continuous $\mathrm{O}_{2}$ therapy tends to reduce but not abolish the occurrence of desaturation, ${ }^{36}$ given the fact that only point checks are made to adjust $\mathrm{O}_{2}$ flow to actual patients' needs.
It has also been demonstrated that the duration of $\mathrm{O}_{2}$ therapy was an independent risk factor for developing postoperative respiratory complications. Patients who require $\mathrm{O}_{2}$ for $\geq 75 \%$ of recovery room time (or greater than $90 \mathrm{~min}$ ) appear to be at greater risk of developing respiratory complications. ${ }^{45}$ This fact may suggest that some patients are not adequately screened for risk factors such as OSA by standard preanaesthesia testing, and that a device dedicated to continuous monitoring of $\mathrm{O}_{2}$ administration (either alarms on duration and flow variations) may help to detect such high-risk patients. The increased resource use in patients with longer oxygen therapy requirement in the recovery room likely reflects the increase in occurrence of pulmonary respiratory complications requiring invasive and non-invasive ventilatory support, especially on the day of surgery.

In L'her et al's study, it was shown that the partial or complete oxygen weaning was significantly increased with automated oxygen titration in comparison with standard oxygen administration, in patients managed in the emergency department during 3 hours. ${ }^{10}$

In the specific setting of postoperative patients, the reduction of the weaning time may improve the efficiency of the turnover of the patients in the recovery room.

\section{Risk and consequences of hyperoxemia}

Few studies have promoted the use of hyperoxia during and after colorectal surgery to reduce wound infection, ${ }^{46}$ but this is not recommended in routine, given controversial data. ${ }^{4-49}$ In a post hoc analysis of the PROXI trial, the authors even pointed out the potential risks of acute coronary syndromes associated with perioperative hyperoxia. ${ }^{50}$ The pathophysiological risks associated with hyperoxia are described for a long time, especially in patients with COPD. ${ }^{4}$ The first recommendation to adjust oxygen flow rates in order to reduce the risks of hyperoxia was published in the early $1960 \mathrm{~s},{ }^{51}$ and several more recent guidelines have reiterated similar recommendations. ${ }^{12}$ The recent demonstration in a large randomised controlled trial (RCT) of an increased mortality for ICU patients assigned to a standard $\mathrm{O}_{2}$ therapy practice, as compared with a more conservative one (absolute risk reduction 0.086 (95\% CI 0.017 to 0.150$)$; $\mathrm{p}=0.01$ ), has revived the debate about potential harm of excessive oxygen therapy in an unselected patients' population. ${ }^{52}$ Adverse effects of hyperoxia could be mediated through a higher oxidative stress, but also increased coronary ${ }^{6}$ and cerebral artery resistances, ${ }^{8}$ all being associated with a potential clinical impact. ${ }^{53}$ Such potential adverse events related to hyperoxia clearly mandates attention in avoiding unnecessary $\mathrm{O}_{2}$ administration. It was demonstrated in two randomised trials from our team ${ }^{10}$ that time with hyperoxia could be reduced with automated oxygen titration, and the hypothesis of the present study is that this problem will also be reduced after thoracic or abdominal surgeries. 


\section{Clinical data with automated oxygen titration}

Several systems have been developed to titrate oxygen flow rate in neonates and in adult patients. ${ }^{10} 14$ In a previous RCT on adult patients admitted to the ED for acute hypoxemic respiratory failure, the use of automated $\mathrm{O}_{2}$ administration was found superior to manual $\mathrm{O}_{2}$ administration to improve the time spent within oxygenation targets, with a between-group difference of $29 \%,{ }^{10}$ as already observed in other studies on $\mathrm{O}_{2}$ automated administration. ${ }^{556}$ In L'Her et al's study, patients experienced less time with hypoxemia and hyperoxia in the FreeO $\mathrm{O}_{2}$ group. ${ }^{10}$ When receiving automated oxygen, partial or complete oxygen weaning was more frequent during initial care, as compared with standard manual $\mathrm{O}_{2}$ administration.

As automated closed-loop $\mathrm{O}_{2}$ administration is not the standard of care for postoperative patients, we did not design this study as a non-inferiority study but rather as a superiority study. One limitation of the study is that investigators are aware of the inclusion group, and blinding is difficult in studies with respiratory support. However, given the characteristics of the two strategies under evaluation, a doubleblind trial was not possible. Second, the assessment of the oxygenation status could be considered as more precise by analysing blood gas sample rather than $\mathrm{SpO}_{2}$. However, this would not enable continuous oxygenation monitoring up to 3 days. Moreover, only continuous of non-averaged $\mathrm{SpO}_{2}$ values do enable precise and rapid adjustments of the $\mathrm{O}_{2}$ flow, in response to exact patients' needs.

Noteworthy, all patients in both groups will be continuously monitored using the same oximeter, which may represent a strength of this study; therefore, the $\mathrm{FreeO}_{2}$ PostOp will also represent the largest prospective study comparing two oxygenation strategies over such a period in the postoperative setting.

In conclusion, the $\mathrm{FreeO}_{2}$ PostOp trial is a pragmatic RCT designed to test the hypothesis that automated closedloop $\mathrm{O}_{2}$ administration is superior to standard manual $\mathrm{O}_{2}$ administration, during the postoperative care of patients with major abdominal or thoracic surgery. To the best of our knowledge, the $\mathrm{FreeO}_{2}$ PostOp trial is the first to evaluate the usefulness of automated closed-loop $\mathrm{O}_{2}$ administration after extubation in such an indication.

\section{Author affiliations}

${ }^{1}$ Medical Intensive Care, CHRU de Brest-La Cavale Blanche, Brest, France ${ }^{2}$ LATIM INSERM UMR 1101, FHU Techsan, Université de Bretagne Occidentale, Brest, France

${ }^{3}$ Intensive Care Unit, Department of Anesthesiology B, DAR B CHU de Montpellier, Hôpital Saint Eloi, Université Montpellier 1, Montpellier, France

${ }^{4}$ Anesthesiology Department, CHRU de Brest-La Cavale Blanche, Brest, France

${ }^{5}$ Anesthesiology Department, Hôpital Estaing, Centre Hospitalier Universitaire

Clermont-Ferrand, Clermont-Ferrand, France

${ }^{6}$ Anesthesiology Department, CHU de Poitiers, Poitiers Cedex, France

${ }^{7}$ R\&D, Oxynov Inc., Technopôle Brest Iroise, Plouzané, France

${ }^{8}$ Research laboratory, Centre de recherche de l'Institut de Cardiologie et de Pneumologie de Québec, Québec, France

${ }^{9}$ Centre d'Investigation Clinique CIC INSERM 1412, CHRU de Brest-La Cavale Blanche, Brest, France
Contributors EL'h and FL designed this study, drafted the manuscript of the protocol and critically revised the manuscript. EL'h, SJ, DV, CJ, BH, EF, TK, VP, $\mathrm{P}-\mathrm{AB}$ and FL participated in the conduct of the study. MG and EN participated in the protocol methodological assessment and statistical plan. All authors read and approved the final manuscript.

Funding This trial was funded by the French Ministry of Health obtained in 2014 from a regional hospital clinical research programme (Programme Hospitalier de Recherche Clinique Interrégional HUG0 2014).

Competing interests El'h reports lecture fees and travel expenses for lectures given at academic meetings from GE Healthcare, Sedana Medical, Smiths Medical, Air Liquide Medical Systems. EL'h and FL are the inventors of the Free02 device and founded Oxynov Inc. to develop the commercial device. The firm Oxynov Inc. provides the automated oxygen therapy equipment to all the participating centres but has no other involvement in the study.

Patient consent Not required.

Ethics approval The Institutional Review Board of the University Hospital of Brest, France (CPP Ouest 6-845) approved the trial for all French centres on 2 February2015 (IDRCB RB14-060). The institutional review board from the Québec Heart and Lung Institute (Canada) approved the trial for their own centre. Any protocol modification will be submitted for review and approval by the ethics committee.

Provenance and peer review Not commissioned; externally peer reviewed.

Data sharing statement All investigators will have access to the final data set. Participant-level data sets will be made accessible on a controlled access basis.

Open access This is an open access article distributed in accordance with the Creative Commons Attribution Non Commercial (CC BY-NC 4.0) license, which permits others to distribute, remix, adapt, build upon this work non-commercially, and license their derivative works on different terms, provided the original work is properly cited, appropriate credit is given, any changes made indicated, and the use is non-commercial. See: http://creativecommons.org/licenses/by-nc/4.0/.

\section{REFERENCES}

1. O'Driscoll BR, Howard LS, Earis J, et al. BTS guideline for oxygen use in adults in healthcare and emergency settings. Thorax 2017;72:ii1-ii90.

2. Committee GS. Global strategy for the diagnosis, maagement, and prevention of chronic obstructive pulmonary disease. 2016. Date http://goldcopd.org/global-strategy-diagnosis-managementprevention-copd-2016 (last accessed 30 Mar 2018).

3. Abdo WF, Heunks LM. Oxygen-induced hypercapnia in COPD: myths and facts. Crit Care 2012;16:323.

4. Aubier M, Murciano D, Milic-Emili J, et al. Effects of the administration of $\mathrm{O} 2$ on ventilation and blood gases in patients with chronic obstructive pulmonary disease during acute respiratory failure. Am Rev Respir Dis 1980;122:747-54.

5. Austin MA, Wills KE, Blizzard L, et al. Effect of high flow oxygen on mortality in chronic obstructive pulmonary disease patients in prehospital setting: randomised controlled trial. BMJ 2010;341:c5462.

6. Wijesinghe M, Perrin K, Ranchord A, et al. Routine use of oxygen in the treatment of myocardial infarction: systematic review. Heart 2009;95:198-202.

7. Farquhar $\mathrm{H}$, Weatherall $\mathrm{M}$, Wijesinghe $\mathrm{M}$, et al. Systematic review of studies of the effect of hyperoxia on coronary blood flow. Am Heart $J$ 2009;158:371-7.

8. Floyd TF, Clark JM, Gelfand R, et al. Independent cerebral vasoconstrictive effects of hyperoxia and accompanying arterial hypocapnia at 1 ATA. J Appl Physiol 2003;95:2453-61.

9. Mok W, Wang W, Cooper S, et al. Attitudes towards vital signs monitoring in the detection of clinical deterioration: scale development and survey of ward nurses. Int J Qual Health Care 2015;27:207-13.

10. L'Her E, Dias P, Gouillou M, et al. Automatic versus manual oxygen administration in the emergency department. Eur Respir $J$ 2017;50:1602552.

11. Claure N, Gerhardt T, Everett R, et al. Closed-loop controlled inspired oxygen concentration for mechanically ventilated very low birth weight infants with frequent episodes of hypoxemia. Pediatrics 2001;107:1120-4.

12. Claure N, Bancalari E, D'Ugard C, et al. Multicenter crossover study of automated control of inspired oxygen in ventilated preterm infants. Pediatrics 2011;127:e76-e83. 
13. Hallenberger A, Poets CF, Horn W, et al. Closed-loop automatic oxygen control (CLAC) in preterm infants: a randomized controlled trial. Pediatrics 2014;133:e379-e385.

14. Lellouche F, L'her E, L'Her E. Automated oxygen flow titration to maintain constant oxygenation. Respir Care 2012;57:1254-62.

15. Lellouche F, Bouchard PA, Roberge M, et al. Automated oxygen titration and weaning with FreeO2 in patients with acute exacerbation of COPD: a pilot randomized trial. Int J Chron Obstruct Pulmon Dis 2016;11:1983-90.

16. Weiser TG, Regenbogen SE, Thompson KD, et al. An estimation of the global volume of surgery: a modelling strategy based on available data. Lancet 2008;372:139-44.

17. Khuri SF, Henderson WG, DePalma RG, et al. Determinants of long-term survival after major surgery and the adverse effect of postoperative complications. Ann Surg 2005;242:32-48.

18. Shander A, Fleisher LA, Barie PS, et al. Clinical and economic burden of postoperative pulmonary complications: patient safety summit on definition, risk-reducing interventions, and preventive strategies. Crit Care Med 2011;39:2163-72.

19. Arozullah AM, Daley J, Henderson WG, et al. Multifactorial risk index for predicting postoperative respiratory failure in men after major noncardiac surgery. The National Veterans Administration Surgical Quality Improvement Program. Ann Surg 2000;232:242-53.

20. Lawrence VA, Dhanda R, Hilsenbeck SG, et al. Risk of pulmonary complications after elective abdominal surgery. Chest 1996;110:744-50.

21. Lindberg P, Gunnarsson L, Tokics L, et al. Atelectasis and lung function in the postoperative period. Acta Anaesthesiol Scand 1992;36:546-53.

22. International council for harmonization of technical requirements for pharmaceuticals for human use. Guidelines for good clinical practice-R2. 2016. Date http://www.ich.org/fileadmin/Public_Web_ Site/ICH_Products/Guidelines/Efficacy/E6/E6_R2_Step_4_2016_ 1109.pdf (last accessed 7 Aug 2018).

23. Mazo V, Sabaté S, Canet J, et al. Prospective external validation of a predictive score for postoperative pulmonary complications. Anesthesiology 2014;121:219-31.

24. Chan AW, Tetzlaff JM, Altman DG, et al. SPIRIT 2013 statement: defining standard protocol items for clinical trials. Ann Intern Med 2013;158:200-7.

25. Schulz KF, Altman DG, Moher D. CONSORT Group. CONSORT 2010 statement: updated guidelines for reporting parallel group randomised trials. BMJ 2010;340:c332.

26. Karcz M, Papadakos PJ. Respiratory complications in the postanesthesia care unit: a review of pathophysiological mechanisms. Can J Respir Ther 2013;49:21-9.

27. Serpa Neto A, Hemmes SN, Barbas CS, et al. Incidence of mortality and morbidity related to postoperative lung injury in patients who have undergone abdominal or thoracic surgery: a systematic review and meta-analysis. Lancet Respir Med 2014;2:1007-15.

28. Bateman NT, Leach RM. ABC of oxygen. Acute oxygen therapy. BMJ 1998;317:798-801.

29. Galatius-Jensen S, Hansen J, Rasmussen V, et al. Nocturnal hypoxaemia after myocardial infarction: association with nocturnal myocardial ischaemia and arrhythmias. Br Heart $J$ 1994;72:23-30.

30. DeWitt DS, Prough DS. Blast-induced brain injury and posttraumatic hypotension and hypoxemia. J Neurotrauma 2009;26:877-87.

31. Selinger SR, Kennedy TP, Buescher P, et al. Effects of removing oxygen from patients with chronic obstructive pulmonary disease. Am Rev Respir Dis 1987;136:85-91.

32. Nattie EE, Doble EA. Threshold of intermittent hypoxia-induced right ventricular hypertrophy in the rat. Respir Physiol 1984;56:253-9.

33. Jones JG, Jordan C, Scudder C, et al. Episodic postoperative oxygen desaturation: the value of added oxygen. $J R$ Soc Med 1985;78:1019-22.

34. Catley DM, Thornton C, Jordan C, et al. Pronounced, episodic oxygen desaturation in the postoperative period: its association with ventilatory pattern and analgesic regimen. Anesthesiology 1985;63:20-8.
35. Gögenur I, Rosenberg-Adamsen S, Lie C, et al. Relationship between nocturnal hypoxaemia, tachycardia and myocardial ischaemia after major abdominal surgery. Br J Anaesth 2004;93:333-8.

36. Canet J, Ricos M, Vidal F. Early postoperative arterial oxygen desaturation. Determining factors and response to oxygen therapy. Anesth Analg 1989;69:207-12.

37. Ramachandran SK, Nafiu OO, Ghaferi A, et al. Independent predictors and outcomes of unanticipated early postoperative tracheal intubation after nonemergent, noncardiac surgery. Anesthesiology 2011;115:44-53.

38. Gögenur I, Wildschiøtz G, Rosenberg J. Circadian distribution of sleep phases after major abdominal surgery. $\mathrm{Br} J$ Anaesth 2008;100:45-9.

39. Chung F, Liao P, Elsaid $\mathrm{H}$, et al. Factors associated with postoperative exacerbation of sleep-disordered breathing. Anesthesiology 2014;120:299-311.

40. Rosenberg J, Wildschiødtz G, Pedersen MH, et al. Late postoperative nocturnal episodic hypoxaemia and associated sleep pattern. Br J Anaesth 1994;72:145-50.

41. Gift AG, Stanik J, Karpenick J, et al. Oxygen saturation in postoperative patients at low risk for hypoxemia: is oxygen therapy needed? Anesth Analg 1995;80:368-72.

42. Pedersen T, Viby-Mogensen J, Ringsted C. Anaesthetic practice and postoperative pulmonary complications. Acta Anaesthesiol Scand 1992;36:812-8.

43. Mathew JP, Rosenbaum SH, O'Connor T, et al. Emergency tracheal intubation in the postanesthesia care unit: physician error or patient disease? Anesth Analg 1990;71:691???697-7.

44. Smetana GW, Lawrence VA, Cornell JE, et al. Preoperative pulmonary risk stratification for noncardiothoracic surgery: systematic review for the American College of Physicians. Ann Intern Med 2006;144:581-95.

45. Ramachandran SK, Thompson A, Pandit JJ, et al. Retrospective observational evaluation of postoperative oxygen saturation levels and associated postoperative respiratory complications and hospital resource utilization. PLoS One 2017;12:e0175408.

46. Greif R, Akça O, Horn EP, et al. Supplemental perioperative oxygen to reduce the incidence of surgical-wound infection. N Engl J Med 2000;342:161-7.

47. Meyhoff CS, Wetterslev J, Jorgensen LN, et al. Effect of high perioperative oxygen fraction on surgical site infection and pulmonary complications after abdominal surgery: the PROX randomized clinical trial. JAMA 2009;302:1543-50

48. Togioka B, Galvagno S, Sumida S, et al. The role of perioperative high inspired oxygen therapy in reducing surgical site infection: a meta-analysis. Anesth Analg 2012;114:334-42.

49. Wetterslev J, Meyhoff CS, Jørgensen LN, et al. The effects of high perioperative inspiratory oxygen fraction for adult surgical patients. Cochrane Database Syst Rev 2015;6:CD008884.

50. Fonnes S, Gögenur I, Søndergaard ES, et al. Perioperative hyperoxia-Long-term impact on cardiovascular complications after abdominal surgery, a post hoc analysis of the PROXI trial. Int $J$ Cardiol 2016;215:238-43.

51. Campbell EJ. The J. Burns Amberson Lecture. The management of acute respiratory failure in chronic bronchitis and emphysema. Am Rev Respir Dis 1967;96:626-39.

52. Girardis M, Busani S, Damiani E, et al. Effect of conservative vs conventional oxygen therapy on mortality among patients in an intensive care unit: the oxygen-ICU randomized clinical trial. JAMA 2016;316:1583-9.

53. Stub D, Smith K, Bernard S, et al. Air versus oxygen in ST-segmentelevation myocardial infarction. Circulation 2015;131:2143-50.

54. Damiani E, Adrario E, Girardis M, et al. Arterial hyperoxia and mortality in critically ill patients: a systematic review and metaanalysis. Crit Care 2014;18:711.

55. Cirio S, Nava S. Pilot study of a new device to titrate oxygen flow in hypoxic patients on long-term oxygen therapy. Respir Care 2011;56:429-34.

56. Rice KL, Schmidt MF, Buan JS, et al. AccuO2 oximetry-driven oxygen-conserving device versus fixed-dose oxygen devices in stable COPD patients. Respir Care 2011;56:1901-5. 